\title{
Equation of state of graphite-like BC
}

\author{
Vladimir L. Solozhenko ${ }^{\text {a,* }}$, Oleksandr O. Kurakevych ${ }^{\text {a }}$, Elena G. Solozhenko ${ }^{\text {b }}$ \\ Jiuhua Chen ${ }^{\mathrm{c}}$, John B. Parise ${ }^{\mathrm{c}}$ \\ ${ }^{a}$ LPMTM-CNRS, Université Paris Nord, F-93430 Villetaneuse, France \\ ${ }^{\mathrm{b}}$ Institute for Superhard Materials of the National Academy of Sciences of Ukraine, Kiev 04074, Ukraine \\ ${ }^{\mathrm{c}}$ Mineral Physics Institute and Department of Geosciences, State University of New York at Stony Brook, Stony Brook, NY 11794-2100, USA
}

Received 27 September 2005; accepted 13 November 2005 by C.N.R. Rao

Available online 1 December 2005

\begin{abstract}
The compressibility of turbostratic boron-substituted graphite (t-BC) was measured up to $12 \mathrm{GPa}$ at room temperature using energy-dispersive $\mathrm{X}$-ray powder diffraction with synchrotron radiation. A fit to the experimental $p$ - $V$ data using Birch-Murnaghan equation of state gives values of the t-BC bulk modulus 23(2) GPa and its pressure derivative 8.0(6). These values point to a higher compressibility of t-BC as compared to turbostratic graphite.
\end{abstract}

(C) 2005 Elsevier Ltd. All rights reserved.

PACS: 61.10.Eq; 64; 64.30.+t

Keywords: A. Boron-substituted graphite; C. XRD; E. High pressure

\section{Introduction}

Boron-substituted carbons are oxidation resistant materials and may be used as host phases for intercalation compounds $[1,2]$. These phases are promising precursors for the synthesis of new superhard materials with semiconducting properties. The use of such novel semiconductors could noticeably increase the working temperature of electronic devices. The possibility to synthesize new superhard materials in the B-C system at high pressures and temperatures has been very recently shown by Solozhenko et al. [3]. However, till now no efforts were made to study the high pressure behavior of such materials.

In the present work the $300-\mathrm{K}$ equation of state of turbostratic graphite-like BC (t-BC) has been studied up to 12 GPa using energy-dispersive X-ray powder diffraction with synchrotron radiation.

\footnotetext{
* Corresponding author. Address: LPMTM-CNRS, Institut Galilée, Université Paris Nord, 99, av. J.B. Clément, 93430 Villetaneuse, France. Tel.: +33 1494034 89; fax: + 33149403938 .

E-mail address: vls@1pmtm.univ-paris13.fr (V.L. Solozhenko).
}

0038-1098/\$ - see front matter (C 2005 Elsevier Ltd. All rights reserved. doi:10.1016/j.ssc.2005.11.025

\section{Experimental}

\subsection{The specimen}

A polycrystalline specimen of $\mathrm{t}-\mathrm{BC}$ has been synthesized by thermal chemical vapor deposition according to the method described in Ref. [2]. The combustion elemental analysis and electron microprobe gave a $\mathrm{B} / \mathrm{C}$ ratio of 0.98 . Secondary ion mass spectroscopy showed that the oxygen impurity content is less than 0.4 at.\%.

\subsection{In situ $X$-ray diffraction experiments to $6 \mathrm{GPa}$}

Experiments up to $6 \mathrm{GPa}$ were carried out using a multianvil X-ray system MAX80 at beamline F2.1, HASYLAB-DESY. The experimental set up has been described elsewhere [4]. Energy-dispersive data were collected on a Canberra solid state Ge-detector with fixed Bragg angle $2 \theta=8.69(3)^{\circ}$ using a white beam collimated to $60 \times 100 \mu^{2}$ (vertical by horizontal) and the detector optics with $2 \theta$ acceptance angle of $0.005^{\circ}$, which ensures a high resolution of the observed diffraction patterns. The detector was calibrated using the $\mathrm{K}_{\alpha}$ and $\mathrm{K}_{\beta}$ fluorescence lines of $\mathrm{Cu}, \mathrm{Rb}, \mathrm{Mo}, \mathrm{Ag}, \mathrm{Ba}$, and $\mathrm{Tb}$.

To decrease the deviatoric stress that was generated during 'cold' compression and thus attain quasi-hydrostatic pressure 
condition during equation-of-state measurements, the samples were preannealed at $800 \mathrm{~K}$ and a given pressure for $10 \mathrm{~min}$. The sample pressure was determined from the lattice constant of $\mathrm{NaCl}$ and $\mathrm{hBN}$ using corresponding equations of state $[5,6]$.

The data obtained allowed us to calculate the pressure dependence of the $a$-parameter, while the lines of boron from the pressure medium (boron/epoxy resin cube) render the estimation of the $c$-parameter impossible.

\subsection{In situ X-ray diffraction experiments to $20 \mathrm{GPa}$}

Experiments up to $20 \mathrm{GPa}$ has been performed using the T-cup, a two-stage 6-8 style high-pressure system [7] with a large anvil press SAM85 [8] at the superconductor wiggler beam line X17B of the National Synchrotron Light Source. The second stage of the system consisted of eight $10 \mathrm{~mm}$ edge length cubes of polycrystalline cubic boron nitride transparent to X-rays. Each cube had one corner truncated into a triangular face; the eight truncations thus formed an octahedral cavity in which the $\mathrm{MgO}$ pressure medium was compressed. Pressures at ambient temperature were evaluated using $\mathrm{NaCl}$ equation of state [5].

Energy-dispersive X-ray diffraction data were collected by an intrinsic Ge solid-state detector fixed at $2 \theta=6.69(1)^{\circ}$ with reference to the direct $\mathrm{X}$-ray beam. The multichannel analyzer used in data collection was calibrated with the characteristic decay energies of ${ }^{57} \mathrm{Co},{ }^{129} \mathrm{I}$ and ${ }^{109} \mathrm{Cd}$. With the storage ring operating at $2.5 \mathrm{GeV}$ and $200 \pm 100 \mathrm{~mA}$ and the incident beam collimated by a set of slits to $100 \times 100 \mu \mathrm{m}^{2}$, X-ray diffraction patterns were collected for 3-10 $\mathrm{min}$ in real time.

In this case the pressure dependence of the $c$-parameter has been evaluated, while interference from the lines of $\mathrm{MgO}$ pressure medium have not allowed to estimate lattice parameter $a$.

\section{Results and discussion}

At ambient conditions, the diffraction pattern of t-BC shows broad, about symmetric 001 line and asymmetric 10 line that is typical for turbostratic (one-dimensionally disordered) structures. The only existing model of the boron-substituted carbon structure suggests the lattice consisting of stacked graphite-like layers with C atoms partially substituted for B [2,9] (Fig. 1). The lattice parameters obtained by angle-dispersive X-ray powder diffraction (D5000 Siemens diffractometer) are $a=$ $2.450(2) \AA$ and $c=3.405(2) \AA$. The inter-layer distance corresponding to the $c$-parameter is lower then that of turbostratic graphite synthesized at similar conditions, $c=$ 3.440 (2) $\AA$. At the same time, the mean length of in-plane bonds is $1.415 \AA(a \sqrt{3 / 3})$, which is slightly longer than that in turbostratic graphite $(1.411 \AA$ ), and is attributed to the higher values of $\mathrm{B}-\mathrm{C}$ and $\mathrm{B}-\mathrm{B}$ bonds in comparison with $\mathrm{C}-\mathrm{C}$ bond.

Experimental energy-dispersive patterns are shown in Fig. 2. The disappearance of all 001 lines has been observed above $12 \mathrm{GPa}$ similar to that for other turbostratic graphite-like structures [10], that restricted the pressure range for equationof-state measurements. The line positions have been estimated

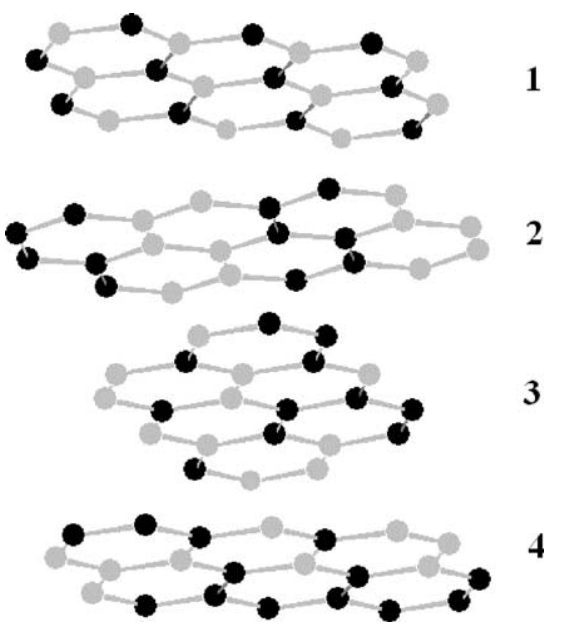

Fig. 1. Representation of the structure of turbostratic graphite-like BC. Black and grey balls correspond to carbon and boron atoms, respectively. Different layers show possible distributions of $\mathrm{B}$ and $\mathrm{C}$ atoms in a graphite-like plane: 1layer built of $\mathrm{B}\left(\mathrm{C}_{3}\right)$ and $\mathrm{C}\left(\mathrm{B}_{3}\right)$ coordination triangles with regular atomic distribution, 2-layer built of $\mathrm{C}\left(\mathrm{BC}_{2}\right)$ and $\mathrm{B}\left(\mathrm{B}_{2} \mathrm{C}\right)$ coordination triangles with regular atomic distribution, 3 and 4-layers with random atomic distribution.

from fitting the experimental reflection profiles to the Pearson and Warren [11] shape functions for 001 and 10 lines, respectively.

Fig. 3 shows the pressure dependence of the lattice parameters $c$ and $a$. The one-dimensional analog of the firstorder Murnaghan equation of state [12] of the form

$\frac{r}{r_{0}}=\left[1+\left(\frac{\beta^{\prime}}{\beta}\right) p\right]^{-1 / \beta^{\prime}}$

was used for approximation of the non-linear relation between normalized lattice parameters and pressure. Here $r$ is the lattice
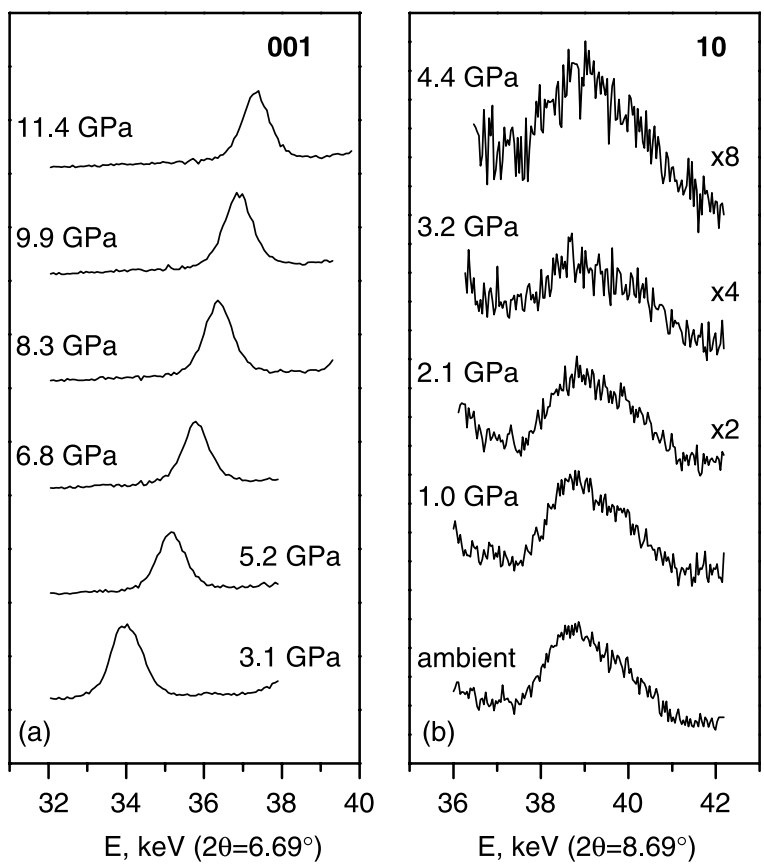

Fig. 2. Experimental energy-dispersive powder diffraction patterns of t-BC obtained using T-cup (a) and MAX80 (b). 

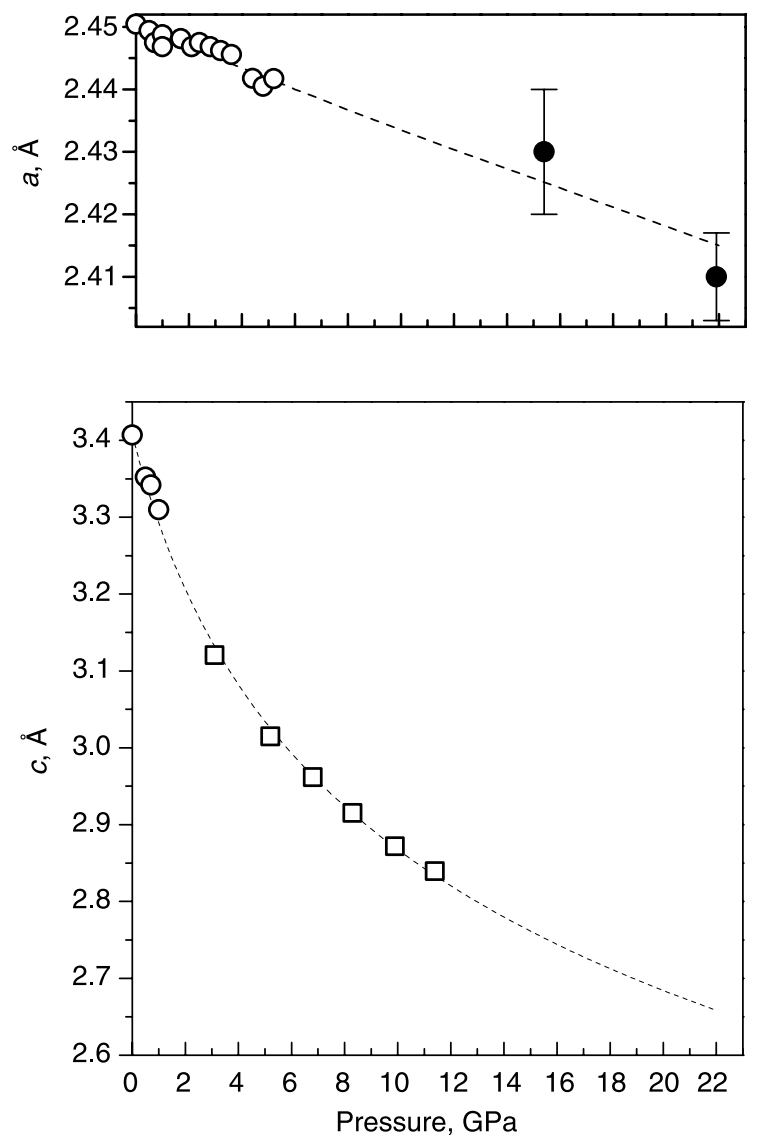

Fig. 3. Lattice parameters of $\mathrm{t}-\mathrm{BC}$ versus pressure at ambient temperature. The dashed line represents the least-squares fit to the experimental data using the first order Murnaghan equation; open circles, solid circles and open squares show the experimental data obtained using MAX80, diamond anvil cell and Tcup, respectively.

parameter (index 0 refers to ambient pressure); $\beta$ is the axial compression modulus, ${ }^{1}$ and $\beta^{\prime}$ is the pressure derivative of $\beta$. The dashed lines in Fig. 3 correspond to Eq. (1) with parameters obtained from a least-squares fit to the experimental data.

Similar to all graphite-like structures, t-BC exhibits much higher compressibility along the $c$-axis than along the $a$-axis as it follows from the $k_{c} / k_{a}=57 \pm 12$ ratio between linear compressibilities in the above directions. The high compressibility along the $c$-axis is demonstrated by the low value of axis modulus $\beta_{c}=25(2) \mathrm{GPa}$ and relatively high value of its pressure derivative $\beta_{c}^{\prime}=8.6(7)$. Within experimental uncertainty, the $a$-axis compression is linear in the pressure range used in this study. Since this phase has a low-compressibility along the $a$-axis, obtaining the reliable value of $\beta_{a}$ requires data over a wide pressure range. In MAX80 experiments we obtained the $a$-parameter values up to $6 \mathrm{GPa}$ only, while two points at higher pressures were obtained in the diamond anvil cell experiments ${ }^{2}$. An average linear compressibility along the

\footnotetext{
${ }^{1}(\beta)^{-1}=k_{r}=-(\mathrm{d} \ln r / \mathrm{d} p)_{p=0}$ is the linear compressibility.

2 The experiments at 15.4 and $21.9 \mathrm{GPa}$ were performed without pressure medium using a membrane-type diamond anvil cell at beamline ID30, ESRF.
}

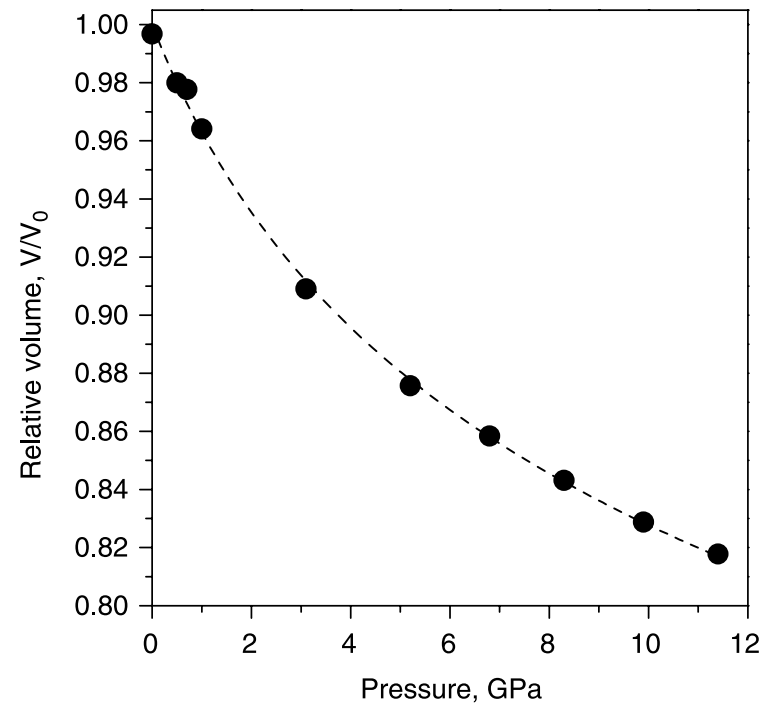

Fig. 4. Relative volume of t-BC versus pressure at ambient temperature. The dashed line represents the least-squares fit to the experimental data using the Birch-Murnaghan equation of state; circles show the experimental data (above $6 \mathrm{GPa} V / V_{0}$-values have been calculated from the experimental values of $c$ parameters and the $a$-parameter values calculated using Eq. (1)).

$a$-axis $\left(\beta_{a}^{\prime}=1\right.$ is the fixed parameter) was, therefore, calculated, yielding an average $a$-axis modulus of $\beta_{a}=$ 1400(200) GPa.

Fig. 4 shows the experimental $p-V$ data for t-BC at $300 \mathrm{~K}$. A least-squares fit to the experimental data using the BirchMurnaghan (third-order Eulerian finite-strain) equation of state [13] gives values of the bulk modulus $B_{0}=23(2) \mathrm{GPa}$ and its pressure derivative $B_{0}^{\prime}=8.0(6)$, with the zero-pressure unit cell volume $V_{0}=17.77(4) \AA^{3}$. The bulk modulus value is less then that of turbostratic graphite $\left(B_{0}=29.5(8) \mathrm{GPa}\right.$, according to Ref. [14]). The higher compressibility of t-BC indicates the strong influence of the in-plane boron on the inter-layer interaction.

\section{Conclusions}

We have measured the change in lattice parameters of turbostratic graphite-like BC up to $12 \mathrm{GPa}$. The ratio between linear coefficients of the t-BC compressibility along the $c$ and $a$ axes is $k_{c} / k_{a}=57 \pm 12$, indicative of a strong anisotropy of bonding forces in the t-BC lattice. A least-squares fit to the volume-pressure data yields bulk modulus $B_{0}=23(2) \mathrm{GPa}$ with corresponding pressure derivative $B_{0}^{\prime}=8.0(6)$.

\section{Acknowledgements}

The authors thank Dr A. Derré for supplying us with the BC sample, Drs M. Vaughan, C. Lathe and D. Andrault for assistance in high-pressure experiments and Dr L.C. Ming for helpful discussions. High-pressure experiments at HASYLAB were supported by the GFZ-Potsdam under the MAX80 program. Part of the experiments was carried out under the General User Proposal No. 4621 at the NSLS which is supported by the US Department of Energy, Division of Materials Sciences and Division of Chemical Sciences under 
Contract No. DE-AC02-98CH10886. This work was partially supported by the National Science Foundation Grant No DMR0452444 and EAR-0510501.

\section{References}

[1] L.E. Jones, P.A. Thrower, Influence of boron on carbon fiber microstructure, physical properties, and oxidation behavior, Carbon 29 (1991) 251-269.

[2] T. Shirasaki, A. Derré, M. Menetrier, A. Tressaud, S. Flandrois, Synthesis and characterization of boron-substituted carbons, Carbon 38 (2000) 1461-1467.

[3] V.L. Solozhenko, N.A. Dubrovinskaia, L.S. Dubrovinsky, Synthesis of bulk superhard semiconducting B-C materials, Appl. Phys. Lett. 85 (2004) 1508-1510.

[4] V.L. Solozhenko, T. Peun, Compression and thermal expansion of hexagonal graphite-like boron nitride up to $7 \mathrm{GPa}$ and $1800 \mathrm{~K}$, J. Phys. Chem. Solids 58 (1997) 1321-1323.

[5] D.L. Decker, Equation of state of sodium chloride, J. Appl. Phys. 37 (1966) 5012-5014.

[6] V.L. Solozhenko, G. Will, F. Elf, Isothermal compression of hexagonal graphite-like boron nitride up to $12 \mathrm{GPa}$, Solid State Commun. 96 (1995) $1-3$.
[7] M.T. Vaughan, D.J. Weidner, Y.B. Wang, et al., T-cup: a new highpressure apparatus for X-ray studies, Rev. High Press. Sci. Technol. 7 (1998) 1520-1522.

[8] D.J. Weidner, M.T. Vaughan, J. Ko, et al., Characterization of stress pressure, and temperature in SAM85, a DIA type high pressure apparatus, in: Y. Syono, M.H. Manghnani (Eds.), High-pressure research: application to earth and planetary sciences, Terra Scientific Publishing Company, AGU, Washington, DC, 1992, pp. 13-17.

[9] B. Ottaviani, A. Derré, E. Grivei, O.A.M. Mahmoud, M.-F. Guimon, S. Flandrois, P. Delhaes, Boronated carbons: structural characterization and low temperature physical properties of disordered solids, J. Mater. Chem. 8 (1998) 197-203.

[10] V.L. Solozhenko, O.O. Kurakevych, On phase transformations of turbostratic phases of the $\mathrm{B}-\mathrm{C}-\mathrm{N}$ system at high pressures and room temperature, J. Superhard Mater. 26 (2004) 35-41.

[11] B.E. Warren, X-ray diffraction in random layer lattices, Phys. Rev. 5 (1941) 693-698.

[12] F.D. Murnaghan, The compressibility of media under extreme pressures, Proc. Natl. Acad. Sci. USA 30 (1944) 244-255.

[13] F. Birch, Finite elastic strain of cubic crystal, Phys. Rev. 71 (1947) 809824.

[14] V.L. Solozhenko, E.G. Solozhenko, On the compressibility of graphite, J. Superhard Mater. 22 (2000) 79-80. 